\title{
The anisotropic hard-sphere crystal-melt interfacial free energy from fluctuations
}

\author{
Ruslan L. Davidchack ${ }^{\mathrm{a})}$ \\ Department of Mathematics, University of Leicester, Leicester LE1 7RH, United Kingdom \\ James R. Morris \\ Metals \& Ceramics Division, Oak Ridge National Laboratory, Oak Ridge, Tennessee 37831-6115 and \\ Materials Science and Engineering, University of Tennessee, Knoxville, Tennessee 37996-2200 \\ Brian B. Laird \\ Department of Chemistry, University of Kansas, Lawrence, Kansas 66045
}

(Received 11 October 2005; accepted 26 July 2006; published online 5 September 2006)

\begin{abstract}
We have calculated the interfacial free energy for the hard-sphere system, as a function of crystal interface orientation, using a method that examines the fluctuations in the height of the interface during molecular dynamics simulations. The approach is particularly sensitive for the anisotropy of the interfacial free energy. We find an average interfacial free energy of $\gamma=0.56 \pm 0.02 k_{B} T \sigma^{-2}$. This value is lower than earlier results based upon direct calculations of the free energy [R. L. Davidchack and B. B. Laird, Phys. Rev. Lett. 85, 4751 (2000)]. However, both the average value and the anisotropy agree with the recent values obtained by extrapolation from direct calculations for a series of the inverse-power potentials [R. L. Davidchack and B. B. Laird, Phys. Rev. Lett. 94, 086102 (2005)]. ㅇ 2006 American Institute of Physics. [DOI: 10.1063/1.2338303]
\end{abstract}

\section{INTRODUCTION}

The crystal-melt interfacial free energy $\gamma$ has been the focus of numerous studies ${ }^{1-16}$ due primarily to its importance in crystal nucleation and growth. ${ }^{17-22}$ Its anisotropy (i.e., the dependence of $\gamma$ on the orientation of the crystal with respect to the interface) is also of particular interest for pattern formation in solidification; for example, the anisotropy can determine the dendrite growth direction in directional solidification. ${ }^{23}$ Even small anisotropies are important, as they are necessary for the stable growth of dendrites. Direct experimental determinations of $\gamma$ are usually based on contact angle measurements. ${ }^{24,25}$ Such measurements are quite difficult and have been done only for a handful of materials. ${ }^{26}$ For most materials, the indirect estimates of this quantity are obtained from the nucleation rate measurements, using the (approximate) relationship between $\gamma$ and the nucleation rate from the classical nucleation theory (or variants thereof). However, since this approach yields an orientationally averaged value of $\gamma$, it is unable to resolve the anisotropy. The lack of reliable direct experimental methods for determining $\gamma$ and its anisotropy has motivated a growing number of studies aimed at computing $\gamma$ and its anisotropy for model systems via molecular simulation. ${ }^{6-16}$

Currently, two qualitatively different approaches are being employed to determine the crystal-melt interfacial free energy in computer simulations. The thermodynamic integration approach stems from the definition of $\gamma$ as the reversible work required to form a unit area of the interface. Within this approach, separate bulk crystal and melt systems prepared at the crystal-melt coexistence conditions are transformed

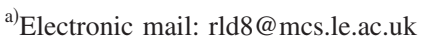

along a continuous path that brings them in contact with each other, creating an interface. Thermodynamic integration is performed along the path in order to determine the reversible work involved in the transformation process. The value of $\gamma$ is then obtained as the work divided by the area of the created interface. Performing the calculation on systems with different crystal orientations can yield information about the free energy anisotropy, provided the results are sufficiently accurate. This approach was first employed by Broughton and Gilmer to calculate the interfacial free energy for a truncated Lennard-Jones potential at the triple point ${ }^{6}$ and was subsequently extended via the cleaving wall method to the systems of hard spheres ${ }^{7}$ and inverse-power potentials ${ }^{15}$ (see Ref. 27 for details).

An alternative approach, the capillary fluctuation method, which examines the magnitude of capillary fluctuations in the profile of a thin strip of the interface, ${ }^{8-12,14,16}$ has been used in the past few years (Fig. 1 illustrates the simulation geometry). For a macroscopically rough interface, the size of the capillary fluctuation modes is related to the interfacial stiffness $\tilde{\gamma}$ by the equipartition theorem as follows: ${ }^{28}$

$$
\left\langle\left|h_{q}\right|^{2}\right\rangle=\frac{k_{B} T}{A \tilde{\gamma} q^{2}},
$$

where $A=b L$ is the area of the flat interface, $q$ is the wave number, and $h_{q}$ is defined in Eq. (3). The interfacial stiffness is given in terms of the interfacial free energy by the formula

$$
\tilde{\gamma}(\theta)=\gamma(\theta)+d^{2} \gamma / d \theta^{2},
$$

where $\theta$ is the angle between the instantaneous local normal to the interface and the average orientation for the reference flat interface. By simulating a number of interfaces with different crystal orientations and measuring the average magni- 


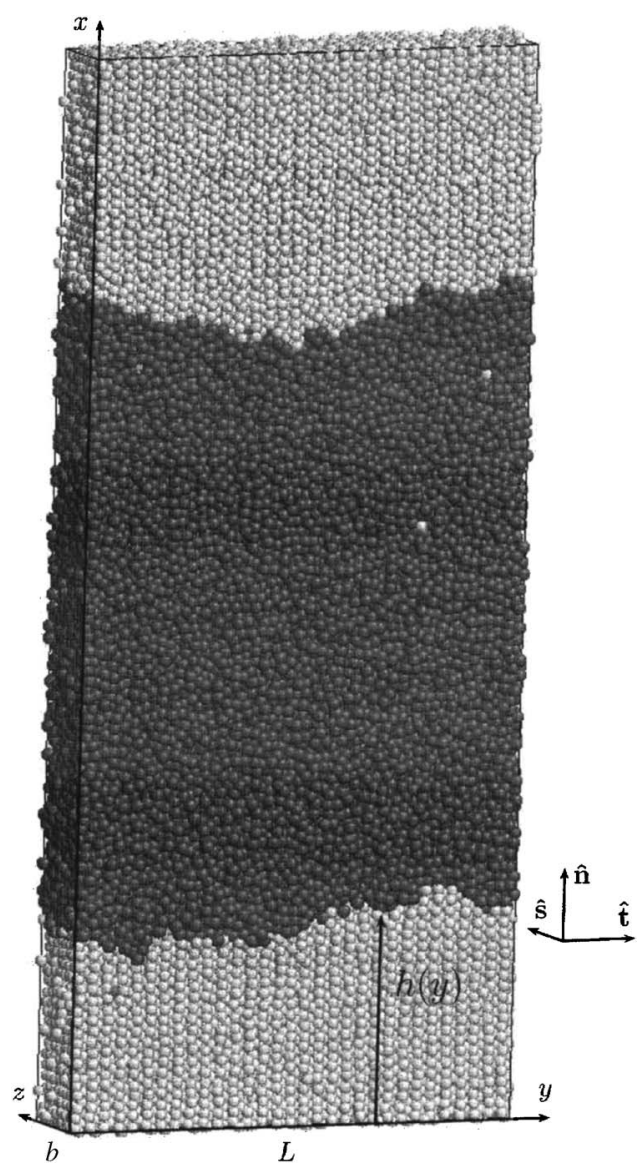

FIG. 1. Sample geometry from the (100) interface simulation. Spheres are colored according to whether they are part of the crystal (light gray) or liquid (dark gray) as determined by the value of the order parameter calculated as described in Ref. 9.

tude of the fluctuation modes, one can use Eq. (1) to determine $\tilde{\gamma}$ from the simulations and Eq. (2) to extract the anisotropic interfacial free energy. This approach has been initially applied to an interatomic potential of $\mathrm{Ni}$ (Ref. 8) and has since been extended to a number of metal and alloy systems. ${ }^{9,14}$ This approach is particularly useful for finding the anisotropy, as the interfacial stiffness is much more sensitive to the small anisotropy in $\gamma$.

Recently, both the thermodynamic integration and the capillary fluctuation approaches were used ${ }^{12,13}$ to calculate the anisotropic free energy of a truncated Lennard-Jones potential. The results were in very good agreement: the difference in the average values was less than $0.3 \%$. The anisotropic free energies were also in good agreement, and both calculations produce the ordering $\gamma_{100}>\gamma_{110}>\gamma_{111}$ for the three principal crystal orientations. This is an excellent test of both approaches: two very different techniques can closely reproduce the same values for the same potential. In addition, the approaches are complementary: the thermodynamic integration approach is a direct method for determining the interfacial free energies, and thus the error bars are smaller for the absolute values, while the fluctuation approach is more sensitive to the differences in the free energies (i.e., anisotropy).

In the present work, we apply the capillary fluctuation method to the hard-sphere (HS) system. As in the Lennard-
Jones case, this provides an independent check on the thermodynamic integration results. In a recent paper ${ }^{15}$ using the thermodynamic integration method the average interfacial free energy was found to be $0.573(5) k_{B} T \sigma^{-2}$, where the number(s) in parentheses represent the estimated error in the last digit(s) shown. This value has been obtained by extrapolation from thermodynamic integration results for a series of inverse-power potentials. It is lower than the earlier estimates of $\gamma=0.62(1) k_{B} T \sigma^{-2}$ obtained by the thermodynamic integration approach applied directly to the HS system ${ }^{7}$ and $0.616(3) k_{B} T \sigma^{-2}$ found in the HS nucleation simulations, ${ }^{21}$ but is closer to $0.55(2) k_{B} T \sigma^{-2}$ estimated from nucleation experiments on hard-sphere-like colloidal systems. ${ }^{4,29}$

The first application of the capillary fluctuation approach to the hard-sphere system was published recently, ${ }^{16}$ which reported the average interfacial free energy $\gamma$ $=0.62(2) k_{B} T \sigma^{-2}$. However, we argue that the author's approach admitted systematic bias leading to the increase in $\gamma$ beyond the reported confidence interval. We present our analysis and comparison of the results in the final section of this article.

We are also interested in a closer examination of the anisotropy. The cleaving calculation of interfacial free energies in the HS systems ${ }^{7}$ has produced the relation $\gamma_{110}$ $>\gamma_{100}>\gamma_{111}$, which is somewhat surprising, since in the Lennard-Jones, as well as other fcc-forming materials, the (100) orientation has the highest free energy. The recent extrapolation results, ${ }^{15}$ as well as those obtained by the capillary fluctuation method, ${ }^{16}$ found the ordering $\gamma_{100}>\gamma_{110}$ $>\gamma_{111}$, which is similar to that in the Lennard-Jones and other fcc-forming systems.

In the calculations reported in this article we obtain values for the average interfacial free energy and anisotropy that are consistent with the extrapolation results ${ }^{15}$ and are lower than previously reported from direct calculation for the HS system. We also obtain a precise estimate of the anisotropy of the interfacial free energy.

\section{COMPUTATIONAL DETAILS}

The hard-sphere molecular dynamics simulation was carried out using the algorithm described in Ref. 30. The systems containing crystal-melt interfaces with different crystal orientations were all prepared and equilibrated at the crystal and melt coexistence densities of $\rho_{c}=1.037 \sigma^{-3}$ and $\rho_{m}=0.939 \sigma^{-3}$, respectively, with an estimated coexistence pressure of $P=11.57(3) k_{B} T \sigma^{-3}$, where $\sigma$ is the sphere diameter. In order to minimize the computational effort and reduce the measurement error, the system geometry is quasitwo-dimensional, with the length of the interfaces $L$ being much larger than the width $b$ (see Fig. 1). The coordinates are chosen such that the $x$ axis is normal to the interface, while the $y$ and $z$ axes correspond to the long and short directions of the interface, respectively. We denote the system orientation with respect to the crystallographic reference frame of the crystal as $(i j k)[\operatorname{lmn}]$, where $(i, j, k) \| \hat{\mathbf{n}}$ is the vector normal to the interface and $(l, m, n) \| \hat{\mathbf{s}}$ is the vector tangent to the interface along the short direction. The tangent vector along the long direction is defined by the cross prod- 
uct $\hat{\mathbf{t}}=\hat{\mathbf{s}} \times \hat{\mathbf{n}}$. The interface profile (averaged over the short direction) is given by the function $x=h(y)$ for each of the two crystal-melt interfaces created due to the periodic boundary conditions. If the values of the function $h(y)$ are measured at a discrete set of points $y_{n}=n \Delta$, where $n=1, \ldots, N$ and $\Delta$ $=L / N$, then the Fourier modes $h_{q}$ in Eq. (1) are defined as follows:

$$
h_{q}=\frac{1}{N} \sum_{n=1}^{N} h\left(y_{n}\right) e^{i q y_{n}},
$$

where the wave number $q$ takes values $q=2 \pi k / L$ with $k$ $=1, \ldots, N$.

To determine the interface profile $h\left(y_{n}\right)$, an order parameter $\phi_{i}$ for each atom $i$ in the system is calculated. The order parameter is defined in such a way that a distinction between local crystal and liquid structures is maximized. As shown in Fig. 1, the crystal and liquid atoms can be identified using the value of the order parameter, and thus outline the location of the crystal-melt interface. In the previous studies employing the fluctuation approach, two types of order parameter have been used. The first one proposed by Hoyt et al. ${ }^{8}$ is calculated using the distances of neighboring atoms from the ideal fcc lattice sites around a given atom $i$,

$$
\phi_{i}=\frac{1}{12} \sum_{j}\left|\mathbf{r}_{i j}-\mathbf{r}_{\mathrm{fcc}}\right|^{2},
$$

where $\mathbf{r}_{i j}=\mathbf{r}_{j}-\mathbf{r}_{i}$ and the sum is over the 12 nearest neighbors.

The second order parameter, proposed by Morris, ${ }^{9}$ uses a set of six reciprocal lattice vectors $\mathbf{q}$ such that $\exp \left(i \mathbf{q} \cdot \mathbf{r}_{\mathrm{fcc}}\right)$ $=1$ to construct the local order parameter for each atom,

$$
\psi_{i}=\left|\frac{1}{6 Z} \sum_{\mathbf{q}} \sum_{j} \exp \left(i \mathbf{q} \cdot \mathbf{r}_{i j}\right)\right|^{2},
$$

where the second sum runs over $Z$ nearest neighbors within the distance $r_{c}$ of a given atom. Essentially, Eq. (5) describes the coherent scattering from an atom and its neighbors, from a set of reciprocal wave vectors. In practice, this order parameter generally works well, but some atoms in the crystal phase have a low value more characteristic of the liquid phase and, less frequently, atoms in the liquid have a larger value more characteristic of the crystal. These mischaracterized atoms are usually isolated, and therefore the discrimination between crystal and liquid structures may be improved by averaging over the neighbors. Thus, the order parameter for each atom is defined as the average,

$$
\phi_{i}=\frac{1}{Z+1}\left(\psi_{i}+\sum_{j} \psi_{j}\right)
$$

In order to ascertain that the final results do not depend on the details of the determination of $h(y)$, we used both methods. The interface location was determined using the atom positions recorded at time intervals of $5.0 \sigma\left(m / k_{B} T\right)^{1 / 2}$ in long molecular dynamics simulation runs. In the first method, the instantaneous atom positions were used, while in the second one we used atom positions averaged over a short time interval of $0.125 \sigma\left(m / k_{B} T\right)^{1 / 2}$, where $m$ is the atom mass.

The interface location at $y_{n}$ is calculated using the order parameters of atoms within a bin centered at $y_{n}$. The two methods also differ in the way this calculation is carried out. In the first method, we use a smooth weighting function $w_{d}(x)$ with compact support of radius $d$ to define the order parameter for all coordinates (not just at the atom positions) as follows:

$$
\phi(x)=\frac{\sum_{i} w_{d}\left(x-x_{i}\right) \phi_{i}}{\sum_{i} w_{d}\left(x-x_{i}\right)},
$$

where $x_{i}$ is the coordinate of atom $i$ and the sums are over atoms within a given bin. We used the weighting function in the form $w_{d}(x)=\left[1-(x / d)^{2}\right]^{2}$ for $|x|<d$ and $w_{d}(x)=0$ otherwise. The interface location $h\left(y_{n}\right)$ is determined from the intersection of $\phi(x)$ with a threshold $\phi_{\mathrm{thr}}$, i.e., $\phi\left(h\left(y_{n}\right)\right)$ $=\phi_{\text {thr. }}$. For sufficiently large weighting function width $d$ and the threshold $\phi_{\text {thr }}$ value chosen in the middle between the typical values of $\phi(x)$ in bulk crystal and melt phases, this equation has a single solution in the vicinity of the crystalmelt interface. In the second method, the interface location $h\left(y_{n}\right)$ is defined as the average position of "interfacial" atoms within a bin centered at $y_{n}$. The atoms are classified as interfacial, if their order parameter $\phi_{i} \in\left[\phi_{\min }, \phi_{\max }\right]$. This method relies on the order parameter of atoms within bulk crystal and liquid phases being strictly outside this range.

In order to determine the stiffness $\tilde{\gamma}$ using Eq. (1), we measure the stiffness of individual modes,

$$
\tilde{\gamma}(q)=\frac{k_{B} T}{b L\left\langle\left|h_{q}\right|^{2}\right\rangle q^{2}} .
$$

Even though Eq. (1) implies that $\widetilde{\gamma}(q)$ should be independent of $q$ within the statistical error, we can expect deviations for both small and large $q$. Since the relaxation time increases with the mode length, ${ }^{11}$ the relaxation times for small $q$ (large wavelength) can be larger than the simulation time, leading to undersampling and therefore, larger uncertainty in the obtained results. For large $q$ (small wavelength) the relationship in Eq. (1) between the stiffness and the average size of the fluctuations, which is based on the assumption of the continuity of the interface profile, is expected to break down when the wavelength becomes commensurate with the crystal lattice spacing. In particular, the value of $\left\langle\left|h_{q}\right|^{2}\right\rangle$ for small amplitude, small wavelength fluctuations will be sensitive to the details of the method for determining the interface location. Based on these considerations, we expect to see a $q$-independent behavior of $\widetilde{\gamma}(q)$ only in a limited range $q$ $\in\left[q_{\min }, q_{\max }\right]$. By applying different methods for locating the interface and varying parameters within these methods, we investigate the sensitivity of $\widetilde{\gamma}(q)$ to the details of the methods and take $q_{\max }$ small enough to exclude data where this sensitivity exceeds the statistical error. The interfacial stiffness $\tilde{\gamma}$ is then obtained as the value of $\widetilde{\gamma}(q)$ averaged over $q$ in the range $\left[q_{\min }, q_{\max }\right]$.

After the stiffnesses for different orientations have been 
TABLE I. Expressions for interfacial free energy and stiffness for different interface orientations from Eq. (10).

\begin{tabular}{ccc}
\hline \hline Interface orientation & $\gamma(\hat{\mathbf{n}}) / \gamma_{0}$ & $\widetilde{\gamma}(\hat{\mathbf{n}}, \hat{\mathbf{t}}) / \gamma_{0}$ \\
\hline$(100)[001]$ & $1+\frac{2}{5} \epsilon_{1}+\frac{4}{7} \epsilon_{2}$ & $1-\frac{18}{5} \epsilon_{1}-\frac{80}{7} \epsilon_{2}$ \\
$(110)[\overline{1} 10]$ & $1-\frac{1}{10} \epsilon_{1}-\frac{13}{14} \epsilon_{2}$ & $1-\frac{21}{10} \epsilon_{1}+\frac{365}{14} \epsilon_{2}$ \\
$(110)[001]$ & $1-\frac{1}{10} \epsilon_{1}-\frac{13}{14} \epsilon_{2}$ & $1+\frac{39}{10} \epsilon_{1}+\frac{155}{14} \epsilon_{2}$ \\
$(111)[\overline{1} 10]$ & $1-\frac{4}{15} \epsilon_{1}+\frac{64}{63} \epsilon_{2}$ & $1+\frac{12}{5} \epsilon_{1}-\frac{1280}{63} \epsilon_{2}$ \\
$(120)[001]$ & $1+\frac{2}{25} \epsilon_{1}-\frac{68}{175} \epsilon_{2}$ & $1+\frac{6}{5} \epsilon_{1}+\frac{104}{35} \epsilon_{2}$ \\
$(120)[\overline{2} 10]$ & $1+\frac{2}{25} \epsilon_{1}-\frac{68}{175} \epsilon_{2}$ & $1-\frac{66}{25} \epsilon_{1}+\frac{88}{7} \epsilon_{2}$ \\
\hline \hline
\end{tabular}

computed, the interfacial free energy and its anisotropy is determined based on the cubic harmonic expansion of Fehlner and Vosko, ${ }^{31}$

$$
\begin{aligned}
\gamma(\mathbf{n}) / \gamma_{0}= & 1+\epsilon_{1}\left(\sum_{i=1}^{3} n_{i}^{4}-\frac{3}{5}\right) \\
& +\epsilon_{2}\left(\sum_{i=1}^{3} n_{i}^{4}+66 n_{1}^{2} n_{2}^{2} n_{3}^{2}-\frac{17}{7}\right),
\end{aligned}
$$

where $\left\{n_{1}, n_{2}, n_{3}\right\}$ are the Cartesian components of the unit vector $\mathbf{n}$ normal to the interfacial plane, $\gamma_{0}$ is the orientationally averaged interfacial free energy and $\epsilon_{1}$ and $\epsilon_{2}$ are expansion coefficients that quantify the anisotropy. A similar expression for the stiffness can be derived using Eq. (2). In particular,

$$
\widetilde{\gamma}(\hat{\mathbf{n}}, \hat{\mathbf{t}})=\left(\gamma(\theta)+\frac{d^{2} \gamma}{d \theta^{2}}\right)_{\theta=0},
$$

where $\gamma(\theta) \equiv \gamma(\hat{\mathbf{n}} \cos \theta+\hat{\mathbf{t}} \sin \theta)$. Note that the stiffness of the interface depends both on the normal $\hat{\mathbf{n}}$ and the tangent $\hat{\mathbf{t}}$ (or $\hat{\mathbf{s}}$ ) direction. For convenience, we provide expressions for $\gamma$ and $\tilde{\gamma}$ for the orientations studied in this work in Table I.

\section{RESULTS}

We have simulated systems containing about $10^{5}$ particles. The sizes of the systems are given in Table II. Before performing production runs we have investigated the dependence of $\tilde{\gamma}(q)$ on various parameters of the capillary fluctuation method. The dependence of $\tilde{\gamma}(q)$ on the choice of the order parameter is shown in Fig. 2. As expected, the stiffness at small $q$ is less sensitive to the type of the order parameter, and the difference in the computed results increases with $q$.

TABLE II. Simulated system sizes and calculated stiffness for different orientations of the hard-sphere crystal-melt interface.

\begin{tabular}{cccc}
\hline \hline Orientation & System size, $\sigma$ & $\tilde{\gamma}, k_{B} T \sigma^{-2}$ & $\tilde{\gamma}$ (fit) \\
\hline$(100)[001]$ & $125.5 \times 128.6 \times 6.273$ & $0.44(3)$ & 0.437 \\
$(110)[\overline{1} 10]$ & $115.3 \times 131.7 \times 6.654$ & $0.42(3)$ & 0.422 \\
$(110)[001]$ & $115.3 \times 139.7 \times 6.273$ & $0.70(3)$ & 0.693 \\
$(111)[\overline{1} 10]$ & $130.4 \times 115.2 \times 6.654$ & $0.67(4)^{\mathrm{a}}$ & 0.696 \\
$(120)[001]$ & $126.2 \times 126.2 \times 6.273$ & $0.59(3)$ & 0.601 \\
$(120)[\overline{2} 10]$ & $112.2 \times 127.0 \times 7.014$ & $0.43(3)$ & 0.427 \\
\hline \hline
\end{tabular}

${ }^{\mathrm{a}}$ This value of stiffness is for the rhcp-crystal-melt, rather than the fcccrystal-melt interface. See text for details.

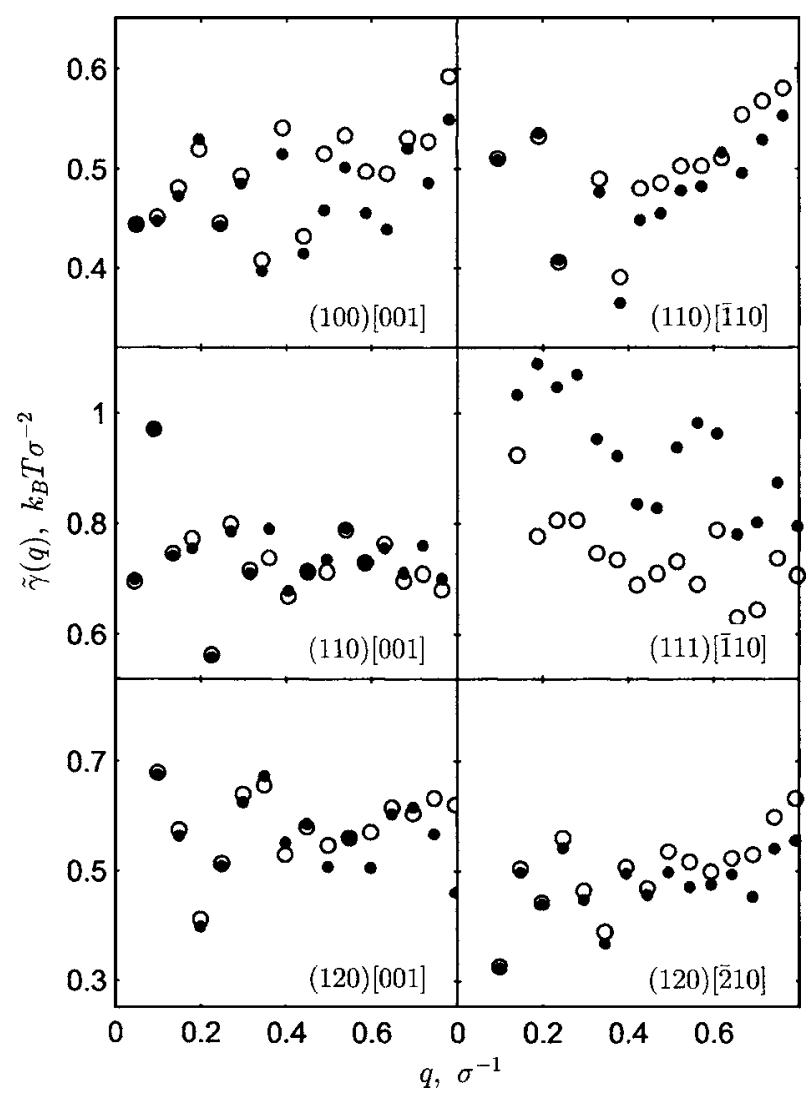

FIG. 2. Stiffness of the fluctuation modes evaluated using different order parameters. Solid dots represent results obtained using Eq. (4), while open circles are computed according to Eqs. (5) and (6). The results are averaged over 200 samples of the interfacial profiles and over the two interfaces in each system.

The exception is the (111)[ $\overline{1} 10]$ orientation, in which the two order parameters produce markedly different results. Closer inspection of the structure of the crystal layers near the fluctuating interface shows that the newly formed layers rarely respect the fcc configuration, but rather form a random hexagonal-close-packed (rhcp) structure through a sequence of stacking faults. This observation is consistent with those discussed in Ref. 11. The order parameter of Hoyt et al. ${ }^{8}$ in Eq. (4), which relies on the proximity of the nearest neighbors to the ideal fcc lattice, tends to mistakenly identify rhcp with the liquid structure (large value of the order parameter), thus leading to erroneously large stiffness values. The order parameter of Morris ${ }^{9}$ correctly identifies rhcp as a crystal phase and therefore is more reliable in calculating stiffness for this orientation. In subsequent calculations, we only used the second order parameter for the (111) orientation and interpret the result as the stiffness of the interface between the rhcp crystal and melt.

Following the analysis carried out in Refs. 32 and 33, we have studied the distributions of interfacial heights and found them to be Gaussian, in agreement with the predictions of the capillary fluctuation theory. We have also investigated the dependence of the results on the threshold $\phi_{\mathrm{thr}}$ and the bin width $\Delta$. Moving the threshold away from the optimal intermediate position leads to a slight decrease in the calculated stiffness. This is consistent with the observations reported in Ref. 16. In addition, our studies have shown that the results 


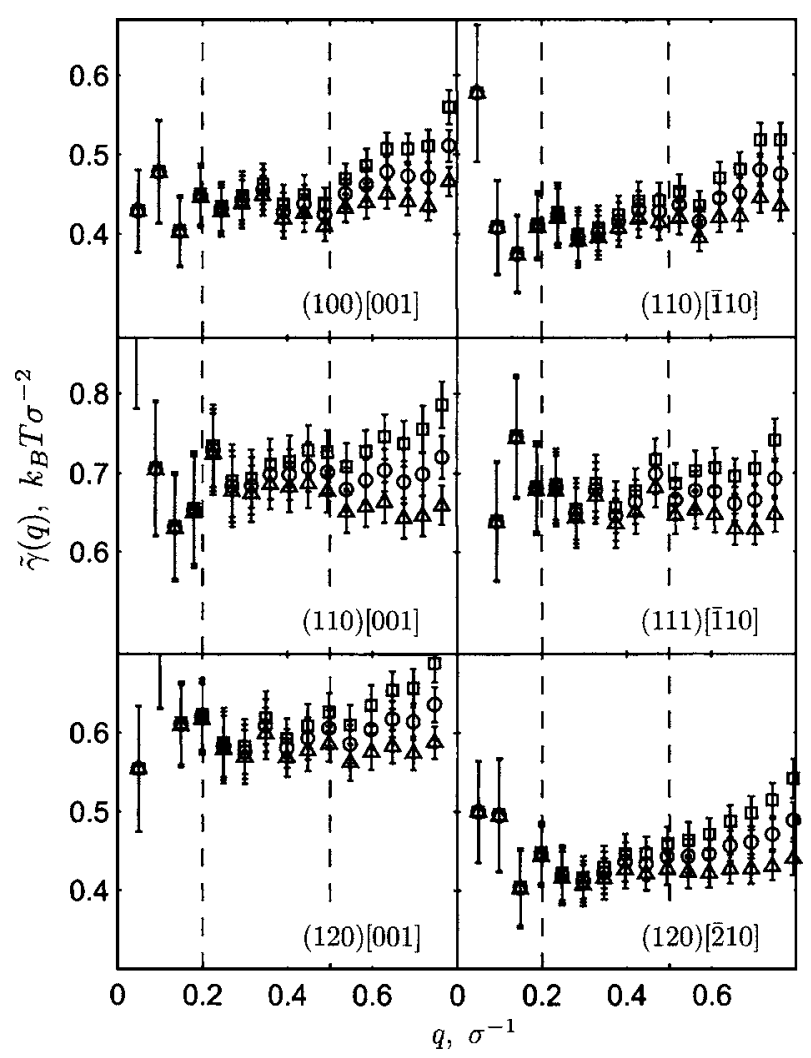

FIG. 3. Stiffness of individual modes $\tilde{\gamma}(q)$ for different orientations of the hard-sphere crystal-melt interface. Triangles, circles, and squares indicate results obtained for bin widths $\Delta$ of $1.5,2.0$, and $2.5 \sigma$, respectively. Error bars indicate the $95 \%$ confidence intervals. Vertical dashed lines show the range of modes $(0.2<q<0.5)$ used to determine the stiffness $\tilde{\gamma}$ in Table II.

are sensitive to the value of the bin width $\Delta$ : with increasing values of $\Delta$, the short-wavelength fluctuations are smoothed out, resulting in higher values of computed stiffnesses $\widetilde{\gamma}(q)$ for larger $q$. In Fig. 3 we plot $\tilde{\gamma}(q)$ for three different values of the bin width. The results are obtained by averaging over 2000 samples of interfacial profiles and over the two interfaces for each orientation. The statistical errors are estimated using the block-averaging technique. ${ }^{34}$ The parameter $d$ in Eq. (7) is fixed at $4.0 \sigma$.

As discussed earlier, the small $q$ values of the calculated stiffness exhibit large variability due to incomplete sampling (large relaxation time) of long-wavelength modes. For $q$ $>0.2$ the results are more systematic, but show increasing sensitivity to the bin width. According to the capillary fluctuation theory, the stiffness of different modes, as defined by Eq. (8), should be independent of $q$. From the results shown in Fig. 3, it appears possible to adjust the bin width parameter and achieve such independence (within statistical errors) for a wide range of $q$ 's for all orientations. However, the optimal choice of $\Delta$ would be different for different orientations and there is no apparent a priori reason to believe that the specific choice of the bin width (or other parameters) that minimizes the dependence of $\widetilde{\gamma}(q)$ on $q$ leads to an accurate value of the interfacial stiffness.

Therefore, in order to limit possible systematic bias in our results, we restrict the averaging to within the interval $0.2<q<0.5$ where all three values of the bin width parameter yield relatively flat $\tilde{\gamma}(q)$ and the difference in the aver- age stiffness for the three measurements is smaller than $0.01 k_{B} T \sigma^{-2}$ for all orientations. The results are presented in Table II. The errors listed in the table combine the statistical error, which is about $0.01 k_{B} T \sigma^{-2}$ for all orientations except (111) [ $\overline{1} 10]$, where it is $0.02 k_{B} T \sigma^{-2}$, and an estimated systematic error of up to $0.02 k_{B} T \sigma^{-2}$ due to the sensitivity of the results to the details of the methods used in the calculation of the interfacial stiffness.

As discussed above, the simulation of the (111) interface produced the stiffness of the interface between the rhcp crystal and liquid rather than between the fcc crystal and liquid. Since the excess free energy of the stacking faults in hardsphere crystals is very small, ${ }^{11}$ the difference between the two interfacial stiffnesses should be relatively small. However, in order to have consistent description of the fcccrystal-melt interface, we have excluded the (111)[110] orientation from the subsequent analysis.

Using stiffness values from the other five orientations, we have applied linear regression and found the following values of the Fehlner and Vosko parameters: $\gamma_{0}$ $=0.559(17) k_{B} T \sigma^{-2}, \epsilon_{1}=0.072(9)$, and $\epsilon_{2}=-0.004(2)$. Using these parameters, we have calculated the fitted values of the stiffness, shown in the last column of Table II, which illustrate good consistency of the obtained results. The predicted stiffness for the (111) orientation of the fcc-crystal-melt interface is slightly larger than that of the rhcp-crystal-melt interface, and we see the largest deviation from the fit for this orientation. In order to check the system size effects, we have simulated systems with larger interfacial widths $(b$ $=10-11 \sigma)$ and smaller lengths $(L=70-80 \sigma)$. The obtained values of $\tilde{\gamma}(q)$ agree within statistical errors with those for other system sizes for all orientations and all $q$, indicating that, as predicted by Eq. (1), the magnitude of the fluctuation modes $\left\langle\left|h_{q}\right|^{2}\right\rangle$ is inversely proportional to the area of the interface.

\section{DISCUSSION}

A comparison of values for the interfacial free energy of hard-sphere crystal-melt interface obtained by different computational techniques is shown in Table III: thermodynamic integration (Refs. 7 and 15), capillary fluctuation (Ref. 16 and this work), and homogeneous nucleation (Ref. 21). Within the thermodynamic integration approach, $\gamma_{100}, \gamma_{110}$, and $\gamma_{111}$ are measured directly, while parameters $\gamma_{0}$ and $\epsilon_{1,2}$ are calculated based on Eq. (10) (see Ref. 13 for details). Within the capillary fluctuations, all parameters in the table are determined from the stiffness measurements, as described in this article. The authors of Ref. 16 obtained the following stiffness values (in units $k_{B} T \sigma^{-2}$ ): 0.55 for (100)[001], 0.71 for (110)[001], and 0.80 for (111)[ [110], which they used to calculate the parameters listed in Table III. In the nucleation simulations of Ref. 21, the authors obtained an average value of the interfacial free energy, which we identify here with parameter $\gamma_{0}$, although, because Ref. 21 uses classical nucleation theory and other approximations to extract an estimate of the orientationally averaged interfacial free energy, the correspondence between this estimate and $\gamma_{0}$ is only approximate. 
TABLE III. Comparison of values for the interfacial free energy of hard-sphere crystal-melt interface obtained by various computational methods.

\begin{tabular}{lccccc}
\hline \hline & Ref.7 & Ref. 21 & Ref. 15 & Ref. 16 & This work \\
\hline$\gamma_{0}$ & $0.617(6)$ & $0.616(3)$ & $0.573(5)$ & $0.62(2)$ & $0.559(17)$ \\
$\epsilon_{1}$ & $0.07(3)$ & & $0.09(4)$ & 0.056 & $0.072(9)$ \\
$\epsilon_{2}$ & $-0.044(12)$ & & $-0.005(8)$ & -0.0073 & $-0.004(2)$ \\
$\gamma_{100}$ & $0.62(1)$ & $0.592(7)$ & $0.64(2)$ & $0.574(17)$ \\
$\gamma_{110}$ & $0.64(1)$ & $0.571(6)$ & $0.62(2)$ & $0.557(17)$ \\
$\gamma_{111}$ & $0.58(1)$ & $0.557(7)$ & $0.61(2)$ & $0.546(16)$ \\
\hline \hline
\end{tabular}

Even though the earlier calculations ${ }^{7,21}$ estimated the average interfacial free energy at about $0.62 k_{B} T \sigma^{-2}$, the more recent calculations with a better control of systematic deviations yield a lower value of around $0.57 k_{B} T \sigma^{-2}$. It is likely that the confidence intervals for the results stated in Ref. 7 were underestimated because some of the systematic errors in the thermodynamic integration approach, which increased the results [especially for the (110) interface], were not accounted for. Note that the difference in the average free energy between the present and the previous calculations is only about $10 \%$. This may seem small, but is important due to the impact on nucleation rates. Within the classical nucleation theory, the energy of the critical nucleus scales as $\gamma^{3}$, and the nucleation rate varies exponentially with this energy. Thus, our recent results would indicate a much faster nucleation rate.

Note that our results are also lower than those obtained recently within the capillary fluctuation method and reported in Ref. 16. We argue that the implementation of the method in Ref. 16 contains a systematic bias that increases the values of the interfacial free energy. The key feature of the approach used in Ref. 16 is that the stiffness, as calculated by the authors, depends on the value of the threshold parameter $\phi_{\mathrm{thr}}$ in such a way that it has a maximum at the intermediate range of the parameter values. The authors determine $\phi_{\mathrm{thr}}$ from the location of this maximum and thus obtain the value for the interfacial stiffness. However, in our analysis above we show that the dependence of the calculated stiffness on the threshold, as well as other parameters of the fluctuation method, is the most prominent at shorter wavelengths (larger $q$ ). The comparison in Fig. 3 shows that, for most orientations, the shorter modes tend to have higher stiffness. Therefore, by including the modes with $q$ of up to $0.8 \sigma^{-1}$ and by choosing the maximum value of stiffness, the authors of Ref. 16 have introduced a bias towards larger stiffness results.

In the present work, our approach was to test the sensitivity of the calculated stiffness values to the details of the method and discard the results as unreliable if this sensitivity was much larger than the statistical uncertainty. Within this approach, the measurements with wave numbers larger than $q_{\max }=0.5 \sigma^{-1}$ were discarded, and the reported uncertainty in the results includes possible bias due to the specific details of the application of the capillary fluctuation method.

To conclude, the capillary fluctuation approach provides a relatively simple way of determining the crystal-melt interfacial free energy and its anisotropy from molecular simulation. However, since the continuum interface model of the capillary fluctuation theory is incompatible with the atomistic nature of molecular simulations, the implementation of this approach requires a procedure for defining a continuous interfacial profile $h(y)$ based on the atomic configuration at the solid-liquid interface. Since such a procedure is not unique, it is likely to generate systematic errors in the measured magnitudes of the interfacial fluctuations, which increase with increasing wave number $q$. Therefore, it is important to investigate the sensitivity to parameters of each specific implementation of the capillary fluctuation approach in every new system under investigation. Further studies are required to understand the nature of the uncertainties of this approach and to find possible ways of their elimination.

\section{ACKNOWLEDGMENTS}

The authors would like to thank Mark Asta for fruitful discussions regarding the formation of stacking faults in simulations of the (111) interface. One of the authors (R.L.D.) gratefully acknowledges support from the EPSRC Grant No. GR/T27105/01, and computing facilities of the University of Leicester Centre for Mathematical Modelling. The research of another author (J.R.M.) has been sponsored by the Division of Materials Sciences and Engineering, Office of Basic Energy Sciences, U.S. Department of Energy under Contract No. DE-AC05-00OR-22725 with UTBattelle. He also acknowledges partial funding from the Department of Energy's Computational Materials Science Network project, "Microstructural Evolution Based on Fundamental Interfacial Properties," and computer time from the Department of Energy's National Energy Research Scientific Computing Center. Another author (B.B.L.) gratefully acknowledges support from the National Science Foundation under Grant No. CHE9970903.

\footnotetext{
${ }^{1}$ F. Spaepen and R. B. Meyer, Scr. Metall. 10, 257 (1976).

${ }^{2}$ W. A. Curtin, Phys. Rev. B 39, 6775 (1989).

${ }^{3}$ D. W. Marr and A. P. Gast, J. Chem. Phys. 99, 2024 (1993).

${ }^{4}$ D. Marr and A. Gast, Langmuir 10, 1348 (1994).

${ }^{5}$ R. Ohnesorge, H. Lowen, and H. Wagner, Phys. Rev. E 50, 4801 (1994).

${ }^{6}$ J. Q. Broughton and G. H. Gilmer, J. Chem. Phys. 84, 5759 (1986).

${ }^{7}$ R. L. Davidchack and B. B. Laird, Phys. Rev. Lett. 85, 4751 (2000).

${ }^{8}$ J. J. Hoyt, M. Asta, and A. Karma, Phys. Rev. Lett. 86, 5530 (2001).

${ }^{9}$ J. R. Morris, Phys. Rev. B 66, 14404 (2002).

${ }^{10}$ M. Asta, J. J. Hoyt, and A. Karma, Phys. Rev. B 66 100101(R) (2002).

${ }^{11}$ J. J. Hoyt and M. Asta, Phys. Rev. B 65, 214106 (2002).

${ }^{12}$ J. R. Morris and X. Song, J. Chem. Phys. 119, 3920 (2003).

${ }^{13}$ R. L. Davidchack and B. B. Laird, J. Chem. Phys. 118, 7651 (2003).

${ }^{14}$ D. Y. Sun, M. Asta, and J. J. Hoyt, Phys. Rev. B 69, 174103 (2004).

${ }^{15}$ R. L. Davidchack and B. B. Laird, Phys. Rev. Lett. 94, 086102 (2005).

${ }^{16}$ Y. Mu, A. Houk, and X. Song, J. Phys. Chem. B 109, 6500 (2005).
} 
${ }^{17}$ W. A. Tiller, The Science of Crystallization: Microscopic Interfacial Phenomena (Cambridge University Press, New York, 1991).

${ }^{18}$ D. Turnbull, J. Appl. Phys. 21, 1022 (1950).

${ }^{19}$ K. F. Kelton, Solid State Phys. 45, 75 (1991).

${ }^{20}$ S. Auer and D. Frenkel, Nature (London) 409, 1020 (2001).

${ }^{21}$ A. Cacciuto, S. Auer, and D. Frenkel, J. Chem. Phys. 119, 7467 (2003).

${ }^{22}$ S. Auer and D. Frenkel, Adv. Polym. Sci. 173, 149 (2005).

${ }^{23}$ J. J. Hoyt, M. Asta, and A. Karma, Mater. Sci. Eng., R. 41, 121 (2003).

${ }^{24}$ M. Glicksman and C. Vold, Acta Metall. 17, 1 (1969).

${ }^{25}$ M. Gunduz and J. D. Hunt, Acta Metall. 33, 1651 (1985).

${ }^{26} \mathrm{~J}$. Howe, Interfaces in Materials (Wiley, New York, 1997).
${ }^{27}$ B. B. Laird and R. L. Davidchack, J. Phys. Chem. B 109, 17802 (2005). ${ }^{28}$ A. Karma, Phys. Rev. E 48, 3441 (1993).

${ }^{29}$ T. Palberg, J. Phys.: Condens. Matter 11, R323 (1999).

${ }^{30}$ D. C. Rapaport, The Art of Molecular Dynamics Simulation (Cambridge University Press, New York, 1995).

${ }^{31}$ W. R. Fehlner and S. H. Vosko, Can. J. Phys. 54, 2159 (1976).

${ }^{32}$ N. Akino, F. Schmid, and M. P. Allen, Phys. Rev. E 63, 041706 (2001).

${ }^{33}$ S. Wolfsheimer, C. Tanase, K. Shundyak, R. van Roij, and T. Schilling, Phys. Rev. E 73, 061703 (2006)

${ }^{34}$ D. Frenkel and B. Smit, Understanding Molecular Simulation: From Algorithms to Applications, 2nd ed. (Academic, San Diego, 2002). 